\title{
El Gavilán a Distancia
}

\author{
Editado por/Edited by: Gabriela Albuja \\ Recibido/Received: 26/01/2014. Aceptado/Accepted: 15/05/2014 \\ Publicado en línea/Published on Web: 10/09/2014
}

\author{
Leandro Quintana \\ Universidad San Francisco de Quito, Colegio de Ciencias Sociales y Humanidades COCISOH \\ Diego de Robles y Vía Interoceánica, Quito, Ecuador \\ Correo electrónico: leandro.quintana@estud.usfq.edu.ec
}

Resumen

Ecuador se une a una nueva generación de innovación tecnológica gracias a la introducción de un avión no tripulado, el llamado UAV-2 “Gavilán”. Los vehículos aéreos no tripulados UAV (por las siglas en ingles de Unmanned Aerial Vehicle) se han convertido en un accesorio de moda en los arsenales militares de un sinnúmero países alrededor del mundo. Adicionalmente, estos artefactos nos dan una mirada del futuro de la aviación comercial y militar. Con el UAV ecuatoriano bautizado como "Gavilán", la Fuerza Aérea Ecuatoriana (FAE) da la bienvenida a un producto autóctono con esperanzas de implementar su producción y futura exportación. La creación de "Gavilán" no solo marca un hito en la historia de la aviación ecuatoriana, si no que a su vez suma a la nación andina a la vanguardia de las tendencias aeronáuticas en la región. La novedad presentada por estos vehículos surge debido a su capacidad ser piloteados remotamente, a su amplio rango de acción, y a sus variadas capacidades operativas. Frente a esta nueva creación surge una pregunta fundamental: ¿Es posible que el Ecuador utilice de forma inapropiada o ilegalmente estas nuevas tecnologías como lo han hecho otras naciones? La respuesta a esta interrogante puede desprenderse de un análisis al récord histórico de actividades de la Fuerza Aérea Ecuatoriana y a la situación de la región latinoamericana en general.

Palabras clave: vehículo aéreo no tripulado, dron, Fuerza Aérea Ecuatoriana, daño colateral.

\section{Abstract}

Ecuador now joins the new generation of technological innovation with the introduction of a new aircraft named UAV-2 "Hawk". The now infamous UAVs (Unmanned Aerial Vehicles) have become a trendy acquirement for both military and civilian arsenals in various countries worldwide. Ignoring their recent controversial use with military applications, these artifacts give us a glimpse at the future of commercial and military aviation. With the Ecuadorian UAV dubbed "Gavilán" or "Hawk", the Ecuadorian Air Force (FAE) welcomes a local product with hopes of its future deployment and export. The creation of "Hawk" marks a milestone in the history of Ecuadorian aviation thanks to its domestic production and for the fact of adding the Andean nation to the vanguard of aeronautical trends. These are novelty vehicles given they are remotely controlled, have great range of action, payloads and operational variability. Ecuador is preparing for its recognition as a potential manufacturer of new technologies and in a more negative sense, preparing to join the group of users of these so ill-seen machines in modern days. A main question that arises from this new creation is, will Ecuador use these new technologies inappropriately or even illegally as many other nations have done? It could be a close reality given the operational record of the Ecuadorian Air Force and the regions situation in general.

Palabras clave: Unmanned Aerial Vehicle, drone, Ecuadorian Air Force, collateral damage.

Durante las últimas décadas hemos presenciado un enorme progreso tecnológico al interior de varios aspectos de la vida diaria, y la aviación no se presenta como una excepción dentro de éste proceso. Desde el histórico vuelo de Eric Warsitz en 1939, a bordo del Heinkel He178 (primer avión a turbo-reacción), hasta nuestros días, se ha visto una acelerada evolución y expansión en cuanto al uso de las aeronaves (Bellis, 2013). Hoy por hoy las aeronaves son en promedio más veloces, cubren mayores distancias, cuentan con mayor capacidad de carga, y en ciertos casos no requieren de la acción de un piloto. Los famosos Drones o UAVs (Unmanned Aerial Vehicle) se han convertido en un lugar común que despierta reacciones de fascinación, escepticismo y oposición al interior de varios grupos pertenecientes a las esferas civil y militar. Estos novedosos vehículos pueden llevar a cabo tareas como operaciones militares estratégicas, vigilancia policial y de bomberos. Adicionalmente es posible encontrar aplicaciones de uso civil en general, ya que el público puede acceder a estas tecnologías en forma de pasatiempo, como el aeromodelismo, o inclusive para acceder a servicios de entrega a domicilio, como el propuesto por la empresa Amazon en su proyecto "Amazon Prime Air" (Amazon Planea Usar Drones Para Envíos a Domicilio, 2013).

Es común observar al interior de la información exhibida por medios de comunicación masiva el uso primordial de UAVs con fines militares. Los UAV, dentro de las esferas militares y de inteligencia, cumplen con un rol de intervención al interior de situaciones que se presentan como demasiado peligrosas o inaccesibles para aviones piloteados y de mayor tamaño (Tice, 1991). Las mencionadas aeronaves se han convertido en pieza clave de las operaciones bélicas que implican el ataque a objetivos de alta importancia estratégica. Ejemplo de esto fueron 
los ataques perpetrado sobre el número 3 de Al Qaeda en 2008, Khalid Habibi, y sobre Anwar Al-Awlaki en 2011, conocido superior de la mencionada organización (Gardner, 2011).

El uso de estas aeronaves ha sido fuertemente criticado por múltiples grupos de la sociedad civil, quienes argumentan que la utilización de esta tecnología con fines bélicos genera un alto número de daños colaterales, bajas civiles y transgresiones a espacios aéreos soberanos. Si bien la Agencia Central de Inteligencia (CIA) afirma que el porcentaje de fatalidades civiles en ataques de Drones estadounidenses sería igual al 0\% (Shane, 2011), un sinnúmero de especialistas y corresponsales de los medios afirman que este número fluctúa entre el $5 \%$ y el $20 \%$. Adicional a esto, los UAVs se presentan como mecanismos que facultan y facilitan el ejercicio de atentados contra los derechos humanos y el derecho internacional. Según Amnesty International, se han encontrado casos en que los Drones se han convertido en un arma utilizada para llevar a cabo ejecuciones extrajudiciales, virtualmente en cualquier localidad mundial (Amnesty International).

Con el nacimiento de "Gavilán" en los hangares de la Fuerza Aérea Ecuatoriana (FAE), el Ecuador se suma al muy exclusivo grupo de usuarios de estos controversiales vehículos. Sin embargo, este proceso puede degenerar también en la producción de actos controversiales tales como invasión de espacios aéreos soberanos y ataques e incursiones en territorios extranjeros, etc. Se prevé que durante el 2014 se construirán al menos 4 aeronaves no tripuladas UAV-2 “Gavilán” para uso nacional (Correa presenta el primer UAV construido por la Fuerza Aérea Ecuatoriana, 2014).

Dentro de la esfera civil, el UAV-2 se pudiese utilizar para vigilancia de áreas urbanas, rurales, remotas y fronterizas; control de fauna silvestre y ganadería; servicios geológicos y una amplia gama de actividades variadas. Esta aeronave contará con un sistema que le otorga siete horas de autonomía, rastreo satelital y operación totalmente a distancia. Se esperaría que esta tecnología tan avanzada se presente como una alternativa que mejore la seguridad ciudadana por su capacidad de vigilancia remota, de videograbación en alta definición y por otras capacidades de reconocimiento. "Gavilán" se muestra como un hito histórico para la FAE, las Fuerzas Armadas y para el desarrollo tecnológico local. El UAV-2 representa clara evidencia del progreso que se está realizando a nivel local en temas científicos, un factor que es un claro motivo de orgullo. Sin embargo, estos Drones pueden llegar a ser arma de doble filo según su utilización.

Hemos visto que la FAE ha tenido varios problemas en la última década: varios accidentes notables y varios casos de compra irresponsable de aeronaves. Tomando en cuenta estos antecedentes, pudiésemos esperar que estos nuevos vehículos siguiesen esta línea de contratiempos. El UAV abre una nueva dimensión en términos de lo que es posible hacer y lo que no es posible hacer en tiempos de guerra y de paz. Un Dron puede ser un activo muy útil, pero al mismo momento un activo muy peligroso. La habilidad de ser controlado remotamente significa la desaparición de limitaciones que antes eran significantes para la aviación bélica y civil. Ahora el único riesgo que corre alguna institución aeronáutica es de naturaleza económica, ya que estos aparatos suelen ser costosos tanto en fabricación como en su uso.
Los límites morales también se ven afectados por estos vehículos. Se argumentaría que se pierde aspectos de integridad y honor al momento de luchar, y el uso de una maquina no tripulada contra un grupo de seres humanos pudiese ser injusto. Estamos conscientes de la inestabilidad en la frontera norte con el conflicto entre fuerzas nacionales y guerrillas, y los UAVs pudiesen surgir como una nueva arma para su aplicación en estas regiones de tensión. Estos vehículos ahora son una nueva y novedosa arma la cual nos habilita a librar la guerra como nunca se ha visto antes. Podemos llevar a cabo ataques dentro de territorio extranjero desde nuestras propias bases nacionales, ataques que resultarían en desenlaces bélicos exitosos. Por ejemplo, el ataque a Angostura se pudiese repetir con un grupo de UAVs fácilmente del lado ecuatoriano al colombiano o viceversa, minimizando así el número de bajas y pérdidas materiales.

Se pudiese argumentar también en favor de los UAV, ya que algunos proclaman que, en sí, un UAV minimizaría la pérdida de vidas inocentes tanto como de los civiles y los mismos pilotos (Carroll, 2012). Los riesgos físicos a los pilotos son nulos, y esto podría nublar el juicio de aquellos que dirigen a la aeronave desde su computadora. Los objetivos cambiaron, las misiones cambiaron también. El Dron es ahora el responsable de actos llevados a cabo de forma ilícita y/o inmoral como vemos hoy en día, y el hecho mismo de ser un UAV tiende a justificar dichas acciones. Se utiliza esta maquinaria para atacar objetivos estratégicos desde puntos remotos, cuales pueden ser desde bases militares en un país y los objetivos en otro. Los ataques que realizan los EE.UU sobre territorio paquistaní fueron realizados desde bases militares en Afganistán, cual es una violación al derecho internacional de los espacios aéreos soberanos.

El Ecuador ahora tiene una enorme responsabilidad en cuanto se refiere al uso de su nuevo Dron, y debe estar muy al tanto de las consecuencias que su uso puede traer, sobre todo con la opinión pública doméstica y de los grupos extranjeros. Dado su récord previo, la Fuerza Aérea Ecuatoriana debe tener mucho cuidado al utilizar estas aeronaves. Debe tener cuidado en dónde se los utilizaría, con qué propósito se los utilizaría y cómo debe utilizarlos sin poner en riesgo la integridad física y mental de la población local y de no violar soberanía extranjera. Los hermanos Wright, o el brasileño Alberto Santos-Dumont, como padres de la aviación moderna, quedarían sorprendidos con el águila a distancia. Sin embargo ¿ganaría su fascinación, o su decepción? Santos-Dumont cegó su propia vida al observar que sus amadas aeronaves se habían convertido en máquinas de destrucción sin precedentes. ¿Qué pensarían los estadounidenses Orville y Wilbur Wright, quienes tras varios años de experimentos solo consiguieron 4 vuelos cortos exitosos en 1903 , si viesen un avión que vuela sin un piloto humano? El águila a distancia nos muestra el estado actual de las ciencias aeronáuticas, y nos da una ventana al futuro. Lo que nos espera en las próximas décadas de vehículos aéreos ciertamente es emocionante, pero siguiendo la tendencia de aplicar lo nuevo a lo destructivo tal vez ese futuro no sea tan atractivo.

\section{Referencias}

Amazon Planea Usar Drones Para Envíos a Domicilio. (2 de Diciembre de 2013). Recuperado el 20 de Febrero de 2014, 
de Expansion: http://www.expansion.com/2013/12/02/empresas/digitech/1385954678.html

Amnesty International. (s.f.). Drones. Recuperado el 20 de Febrero de 2014, de Amnesty International USA: http://www. amnestyusa.org/our-work/issues/security-and-human-rights/drones

Bellis, M. (20 de Diciembre de 2013). The History of the Jet Engine - Sir Frank Whittle - Hans Von Ohain. Recuperado el 12 de Febrero de 2014, de About.com Inventors: http:// inventors.about.com/library/inventors/

Carroll, R. (2 de Agosto de 2012). The Philosopher Making the Moral Case for US Drones. Recuperado el 20 de Febrero de 2014, de The Guardian: http://www.theguardian.com/ world/2012/aug/02/philosopher-moral-case-drones

Correa presenta el primer UAV construido por la Fuerza Aérea Ecuatoriana. (14 de Enero de 2014). Recuperado el 12 de Febrero de 2014, de defensa.com: http://bit.ly/1oQc57K

Gardner, F. (30 de Septiembre de 2011). Islamist cleric Anwar al-Awlaki killed in Yemen. Recuperado el 12 de Febrero de 2014, de BBC News: http://www.bbc.co.uk/news/world-middle-east-15121879

Shane, S. (11 de Agosto de 2011). C.I.A. Is Disputed On Civilian Toll In Drone Strikes. Recuperado el 20 de Febrero de 2014, de The New York Times: http://www.nytimes. com/2011/08/12/world/asia/12drones.html?pagewanted=a$11 \&$ r $=0$

Tice, B. (1991). Unmanned Aerial Vehicles - The Force Multiplier of the 1990s. Airpower Journal. 